\title{
Differentiation of heterozygotes in recessive albinism
}

\author{
D F ROBERTS*, J G R KROMBERG $\dagger$, AND T JENKINS $\dagger$ \\ From *the Department of Human Genetics, University of Newcastle upon Tyne, England; and the \\ Department of Human Genetics, University of the Witwatersrand, Johannesburg, South Africa.
}

SUMMARY Skin pigmentation was measured by reflectance spectrophotometry in 43 albinos, 44 of their relatives, and 123 random controls among Swazi subjects in southern Africa. Mean reflectance of the subject groups diminishes in the sequence affected subjects, obligate heterozygotes, other unaffected family members, random subjects, and the differences among the unaffected groups are highly significant. A discriminant function fitted to the obligate heterozygotes and random subjects is significant statistically but is not efficient enough to be applied in the diagnosis of the non-carrier state.

Recessive albinism was one of the four disorders identified as inborn errors of metabolism by Garrod in $1908 .^{1}$ That this apparently simple Mendelian disorder is genetically heterogeneous and that there is more than one autosomal locus responsible for it, in addition to that on the $\mathrm{X}$ chromosome, is indicated by the raised rate of parental consanguinity, ${ }^{2}$ informative families, ${ }^{3}$ and biochemical heterogeneity. ${ }^{4}$ The basic defect is a block in the metabolic. pathway of tyrosine due to trypsinase deficiency in melanocytes, though in some forms the biochemical lesions appear to lie elsewhere in the sequence. ${ }^{6}$

Little attention has been given to the identification of heterozygotes. Waardenburg ${ }^{7}$ and Froggatt ${ }^{8}$ suggested that heterozygotes have abnormal translucency of the irides. In the tyrosinase positive form, Zipkin et al ${ }^{9}$ found no differences in parotid salivary tyrosine levels between obligate heterozygotes and normal subjects. In the tyrosinase negative form, van Dorp et $a^{10}$ found overlap in tyrosinase assays between heterozygotes, controls, and affected, so they concluded that it was not possible to detect heterozygotes for this form. Perhaps because in the north-west European context skin colour of albinos appears little different from that of fair skinned normals, it is not surprising that little attention has been given to their differentiation phenotypically. To enquire whether skin colour in heterozygotes differed from the normal homozygote it seemed sensible to look to a dark skinned normal population. This study was therefore carried out in southern Africa where albinism is two to three times as frequent in South

Received for publication 2 March 1985.

Revised version accepted for publication 15 June 1985.
African negro populations as in whites in Europe, with a prevalence of 1 in 3900 in Johannesburg negroes ${ }^{11}$ compared with 1 in 10000 in Northern Ireland. ${ }^{8}$ The subjects were Swazi albinos and their families living in Swaziland itself and in Soweto, Johannesburg, South Africa.

\section{Methods}

For logistical reasons it was not possible to investigate biochemically the subjects in the sample. But in South African negro populations only about $10 \%$ of albinos are tyrosinase negative, ${ }^{12}$ and all the subjects visually and on clinical grounds appeared to be tyrosinase positive.

In Swaziland, announcements in the newspapers and on the radio requested albinos and their families to attend clinics on particular days, while albinos in Soweto, who were already known to the Department of Human Genetics, School of Pathology, The South African Institute for Medical Research and University of the Witwatersrand, were invited to participate. Skin colour was measured by a portable EEL reflectance spectrophotometer, reflectance being measured at nine wavelengths $(426,465,485$, $515,545,575,595,655$, and $685 \mathrm{~nm}$ ). The site of measurement was at the mid-point on the inner surface of the upper arm. For comparison, normal subjects from families in which there was no albinism were invited to participate. Altogether, the male sample consisted of 26 albinos, six fathers of albinos and therefore obligate heterozygotes, 10 unaffected members of their families, and 47 random subjects. For the females there were 17 albinos, 18 mothers of albinos, 10 unaffected female members of their families, and 76 random females. 


\section{Results}

For comparison with other published data, the means and standard deviations of the reflectance at each wavelength are set out in table 1 . Inspection of the means (figure) shows (1) the gross excess reflectance in albinos; (2) the decreasing reflectance in males at each wavelength in the sequence albinos, fathers of albinos, other unaffected relatives, and random males; (3) the same trend in females though to a less pronounced extent, and not at wavelengths 485 and 515, where the mothers and unaffected relatives are interchanged; (4) the dip in the reflectance curve at approximately $550 \mathrm{~nm}$ in albinos, but at $480 \mathrm{~nm}$ in unaffected subjects, both male and female; there is also (5) a slightly increased standard deviation at almost every wavelength of unaffected family members by comparison with parents and random subjects. Examination of the form of the distributions, however, showed pronounced skewness at each wavelength, so the analysis was carried out on logarithmic transformations.

There is a highly significant difference between sexes at each wavelength except $426 \mathrm{~nm}$ where $\mathrm{p}$ is only $0 \cdot 053$. Analysis of variance within and between subject groups was therefore carried out within sexes. There was no significant interaction between sex and subject group except at wavelengths 575 and $685 \mathrm{~nm}$.

When, at each wavelength, the deviations from the log mean are plotted in the sequence of probable gene number (albinos, obligate heterozygotes, other unaffected members of albino families, and random unrelated) it is seen (table 2) that in each case the largest positive deviation (that is, the greatest reflectance and therefore the lightest skins) occurs in the albinos, the greatest negative deviation occurs in the random series, and the parents and other unaffecteds fall between them, in each case the obligate heterozygote being lighter than the other unaffecteds. That is to say, at every wavelength there is the progression from light to dark in the sequence: albino, obligate heterozygote, unaffected family member, unrelated random. This is particularly pronounced in the male series. In females, though the sub-sample means seem to differ to a lesser degree, again the largest positive and largest negative deviations occur in the albinos and in the random series respectively, and the mothers and unaffected family members generally lie between them. At six wavelengths the sequence is the same as in the males, but at two of the wavelengths the mothers and other unaffected members change position, and at one wavelength the unaffecteds show the same deviation as in the randoms. In general, therefore, the pattern is similar in both sexes, but less pronounced in females. To establish whether the obligate heterozygotes, other unaffected members of albino families, and random unrelated subjects differ, analysis of variance within sexes was carried out omitting the albinos who are so obviously different. The three subject groups show highly significant differences in mean reflectance at wavelengths 426 and 685 , and significant differences at $465,515,545$, and 575 .

The differences in skin colour and the fact that the albino gene appears to lighten the skin colour may present the heterozygotes with a social advantage. Schapera ${ }^{13}$ noted that, among the Bakgatla (a sub-

TABLE 1 Percentage reflectance at nine wavelengths.

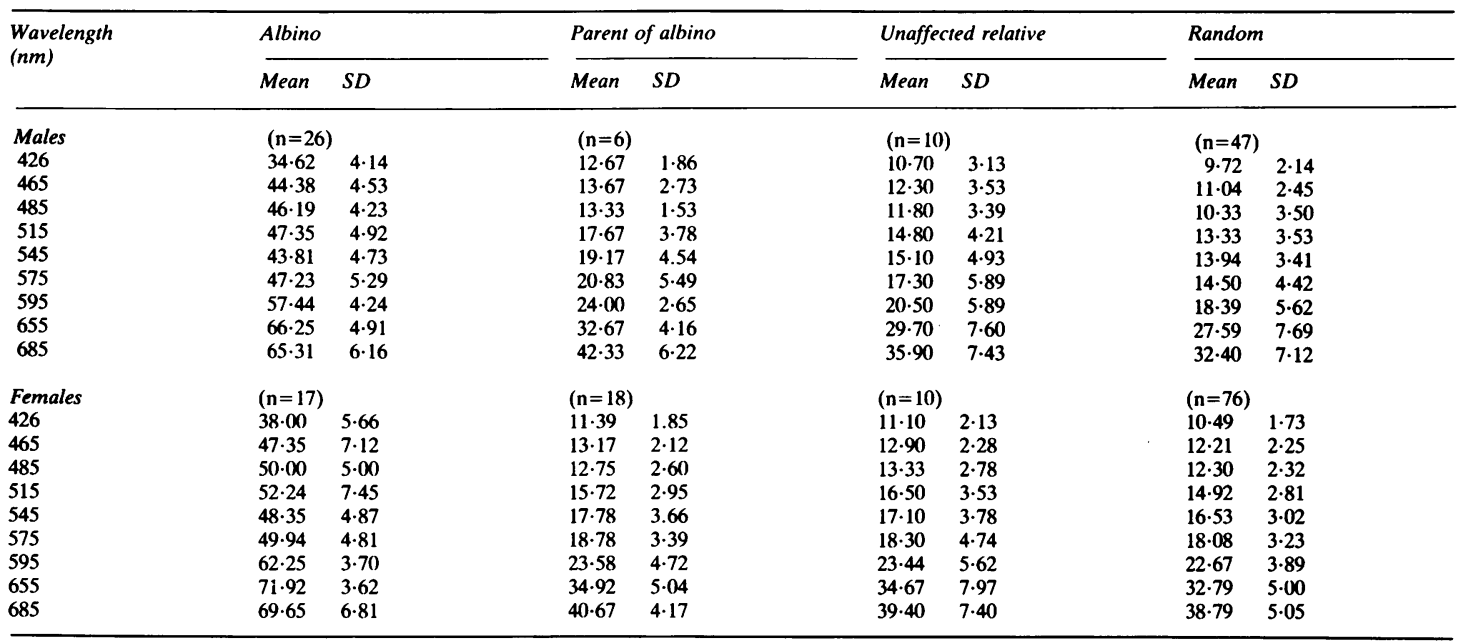




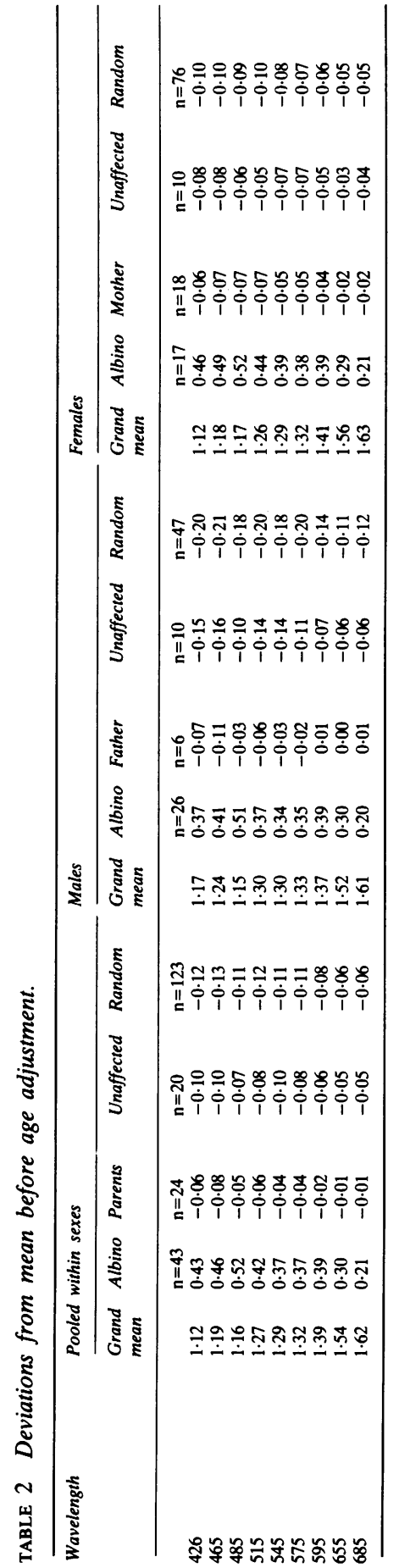

group of the Tswana) of Botswana, the "lightskinned girl of somewhat heavy build" was the generally admired type, who was preferentially selected for a marriage partner. Similarly, lighter skinned people were admired by the Swazi. ${ }^{14}$ The genes for albinism, especially tyrosinase positive, are very common in both the Swazi and the Tswana populations, among whom about 1 in 26 are heterozygotes. ${ }^{11}$ It is therefore possible that such a gene, which is associated with a light skin colour, confers some advantage to the heterozygotes, in terms of selective mating over many generations. Such heterozygote advantage if accompanied by earlier marriage and more children would partly explain the high prevalence of albinism in a tropical climate where albinism would appear to be so obviously detrimental to health.

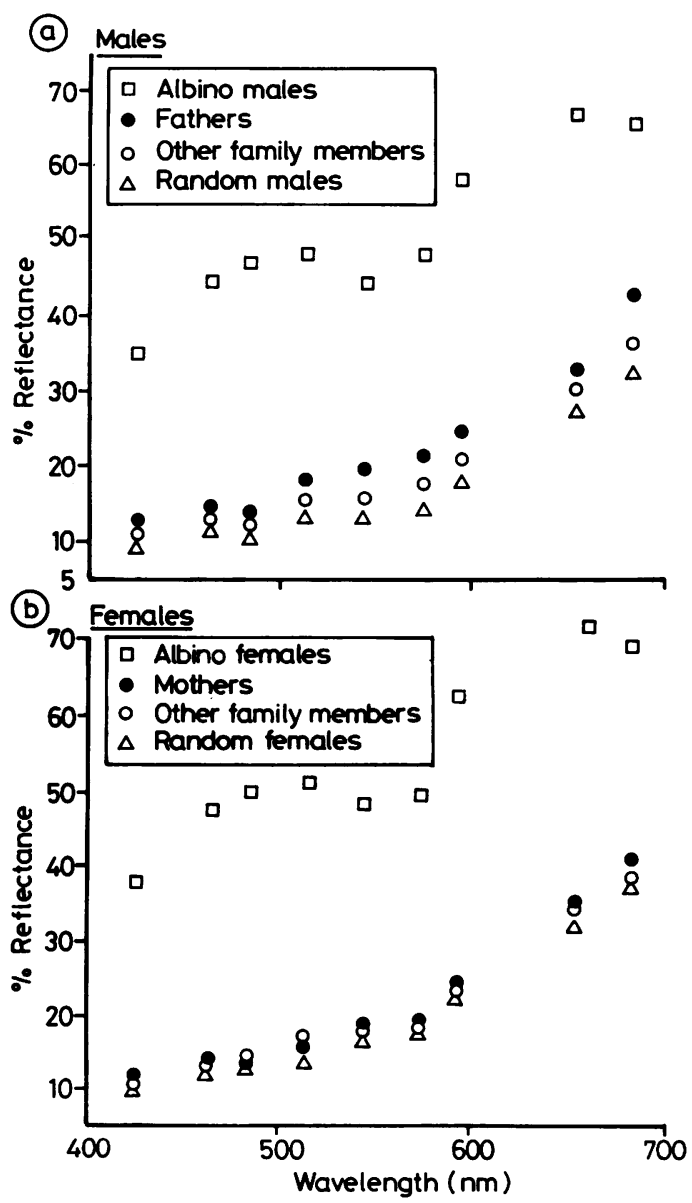

FIGURE Mean reflectance at each wavelength in albinos, their relatives, and unrelated controls. 
There is normally an association of skin reflectance with age, due in part to diminution in the number of melanocytes 1516 in Europeans at estimated rates of 11 to $20 \%$ per 10 years. In the present series also the skin tends to become lighter in unaffected subjects, the regression being significant overall at wavelengths 426,465 , and 541 ; in males at wavelengths $426,465,515,545,575,595$, and 685 $\mathrm{nm}$, but not attaining significance in females. This age effect was removed by covariance analysis, and at each wavelength the deviations adjusted for age were examined. They are very similar to and their sequence remains the same as in the unadjusted material. Again excluding the albinos, comparison was made of the subject group means adjusted for age. Overall, these differences were significant at all wavelengths except 485,595 , and $655 \mathrm{~nm}$; in males, they were highly significant at every wavelength except $655 \mathrm{~nm}$, but not significant at any wavelength in females.

In view of the significant differences that exist between the three unaffected groups of subjects in males and when both sexes are pooled, an attempt was made at multivariate discriminant analysis. First, the mean sex difference in reflectance at each wavelength was added to the female readings to standardise for sex. Then a discriminant function was calculated to differentiate the obligate heterozygotes and the random series. The function $14 \cdot 85 \mathrm{x}_{1}-3 \cdot 09 \mathrm{x}_{2}-7.97 \mathrm{x}_{4}+10 \cdot 12 \mathrm{x}_{5}-12 \cdot 41 \mathrm{x}_{6}+$ $4.91 x_{9}-22.31$ gives significant discrimination $(p=0.015)$, with 17 of the random subjects falling below the range of the obligate heterozygotes, but eight of the latter were misclassified and 35 of the former $(29 \%)$, so obviously the discriminant is not very sensitive. However, stepwise analysis of the total data (combined sexes) showed more significant discrimination $(p=0.0007)$ using the first wavelength, and this was not improved by the addition of any other. The distribution of the function $13 \cdot 273 x-13 \cdot 191$ showed a clear, but unfortunately again not very extensive, zone of non-overlap, with none of the obligate heterozygotes falling below a value of -1 (range -0.8 to $+2 \cdot 8, M=+0 \cdot 643$ ) but 20 of the random doing so (range -2.9 to +3.5 $M=-0 \cdot 127$ ). Seven of the 24 carriers and 42 of the 122 random were misclassified (34\%). The two groups were less widely separated, giving rise to rather more misclassification.

There is nevertheless the suggestion that in subjects of unknown status a small proportion may be identifiable as non-carriers. Heterogeneity of the unaffected members of albino families is suggested by the slightly though not significantly increased standard deviations over the obligate carriers and the random series, at all except two wavelengths in males and all except one wavelength in females. Applying the second discriminant to these 20 unaffected relatives, without incorporating any prior probabilities, two are classified as heterozygotes. These two are one brother and one half-brother of patients. Three are clearly identified as non-carriers, consisting of a brother, a half-brother, and a half-aunt of a proband. Fifteen remain unclassified. For these 20 unaffected family members, their degree of relationship to the proband allows calculation of the probability that each is a carrier, and hence the expected number of carriers among them. This expected number is $11 \cdot 175$. The identification of only two of them as probable heterozygotes indicates the low efficiency of this discriminant. The implication is that only in extreme cases would it be possible to apply usefully this discriminant function for the diagnosis of the non-carrier state.

\section{Discussion}

Whereas in Europeans it is thought to be possible to distinguish, at least after childhood, between tyrosinase positive and negative patients by visual appraisal, similar distinction has not been documented in Africans and may not be so clear cut. However, the interpretation that all subjects in this study were tyrosinase positive is supported by the homogeneity of the skin reflectance distribution curves, for at no wavelength was there any suggestion of bimodality. An extension of this study to include tyrosinase status is planned.

The numbers of subjects in this study are relatively small but the results are of interest on several counts. First, the differences that are shown to exist between the status groups suggest that the albinism genes in single dose exert an effect, and therefore the possibility remains of detecting carriers by biochemical methods, even though the results to date have not been encouraging. The fact that the skin colour differences are demonstrated on a dark skinned population does not restrict a biochemical search for carrier status to such a population.

In view of the significance of the differences in reflectance between status groups, and the significance of the discriminant function, its relative inefficiency in distinguishing carriers and noncarriers in an unknown group is somewhat disappointing, though not unexpected in view of the considerable range of reflectance that exists in normal subjects. However, it may well be possible to combine differentiation by reflectance with some other differentiating measures, should these become available. The difference in the extent of the variation between the status groups between males and females is difficult to explain. This may simply 
be a function of small sample size, but alternatively it may suggest that an $\mathrm{X}$ linked gene intervenes in the expression of autosomal genes influencing pigmentation.

\section{References}

1 Garrod AE. Croonian lectures. Lancet 1908;ii:1, 73, 142, 214.

${ }^{2}$ Fraser Roberts JA. Human pathological conditions determined by any one of several genes. Nature 1932;130:542-3.

3 Trevor-Roper PD. Albinism. Proc $R$ Soc Med 1963;56:21-4.

${ }^{4}$ Witkop CJ, White JG, Jacoby GA. Evidence for two forms of autosomal recessive albinism in man. In: Proceedings of the 2 nd International Congress of Human Genetics. Rome. Istituto G Mendel, 1963:1064-6.

${ }^{5}$ Kugelman TP, van Scott EJ. Tyrosinase activity in melanocytes of human albinos. $J$ Invest Dermatol 1961;37:73-6.

${ }^{6}$ Witkop CJ. Abnormalities of pigmentation. In: Emery AEH, Rimoin DL, eds. Principles and practice of medical genetics. Edinburgh: Churchill Livingstone, 1983;622-52.

7 Waardenburg PJ. Recognizing heterozygote heredity in ocular and universal albinism by the permeability of the iris to light. Ned Tijdschr Geneeskd 1947;91:1863.

${ }^{8}$ Froggatt P. Albinism in Northern Ireland. Ann Hum Genet 1960;24:213-38.
${ }^{9}$ Zipkin I, Hawkins GR, Mazzarella M. The tyrosine tryptophan and protein content of human parotid saliva. In: Sreenby LM, Meyer J, eds. Salivary glands and their secretions. New York: MacMillan, 1964.

10 van Dorp DB, van Haeringen NJ, Glasius E. Evaluation of hairbulb incubation test and tyrosine assay in the classification of albinism. Ophthal Paediatr Genet 1982;1:189-200.

11 Kromberg JGR, Jenkins T. Prevalence of albinism in the South African Negro. S Afr Med J 1982;61:383-6.

12 Kromberg JGR. A genetic and psychosocial study of albinism in Southern Africa. PhD thesis, University of the Witwatersrand, 1985.

13 Schapera A. Married life in an African tribe. London: Faber \& Faber, 1940.

14 Kuper H. The uniform colour. Johannesburg: Witwatersrand University Press, 1947.

15 Snell RS, Bischitz PG. The melanocytes and melanin in human abdominal wall skin. J Anat 1963;97:361-76.

${ }^{16}$ Quevedo WC, Szabo G, Virks J. Influence of age and UV on the populations of DOPA positive melanocytes in human skin. $J$ Invest Dermatol 1969;52:287-90.

Correspondence and requests for reprints to Professor D F Roberts, Department of Human Genetics, University of Newcastle upon Tyne, 19 Claremont Place, Newcastle upon Tyne NE2 4AA. 